\title{
Monitoring of cytomegalovirus (CMV) infection in solid organ transplant recipients: quantitation of CMV DNAemia by two real-time polymerase chain reaction assays
}

\author{
Angela Chiereghin, ${ }^{1}$ Giulia Piccirilli, ${ }^{1}$ Gabriele Turello, ${ }^{1}$ Diego Squarzoni, ${ }^{1}$ Claudia Pavia, ${ }^{1}$ \\ Liliana Gabrielli, ${ }^{1}$ Maria Paola Landini, ${ }^{2}$ Tiziana Lazzarotto \\ ${ }^{1}$ Operative Unit of Clinical Microbiology, Laboratory of Virology, St. Orsola-Malpighi University \\ Hospital, University of Bologna; ${ }^{2}$ Department of Specialised, Experimental, and Diagnostic \\ Medicine, Operative Unit of Clinical Microbiology, Laboratory of Virology, St. Orsola-Malpighi \\ University Hospital, University of Bologna, Italy
}

\section{Summary}

Background and aim: Quantification of cytomegalovirus (CMV) DNAemia is essential in clinical management of post-transplant infection. We evaluated the performances of two quantitative real-time polymerase chain reaction (PCR) assays.

Materials and Methods: 114 serial whole blood samples collected from 14 actively infected transplant recipients were processed by Abbott RealTime CMV PCR kit (Abbott Molecular) and CMV ELITe

Correspondence: Angela Chiereghin, Operative Unit of Clinical Microbiology, Laboratory of Virology, St. Orsola-Malpighi University Hospital, Via Massarenti 9, 40138 Bologna, Italy.

Tel.: +39.051.2144645. Fax: +39.051.307397.

E-mail: angela.chiereghin@gmail.com

Key words: Solid organ transplant; CMV infection; RealTime PCR assay; Whole blood.

Acknowledgements: the authors would like to thank our Linguistic Consultant, Lucy Scioscia, for editing the English language text.

Contributions: $\mathrm{AC}$ acquired and analysed data as well as drafted the paper; GP, LG, GT, DS and CP contributed to the acquisition and analysis of the data; MPL performed a critical revision of the article; TL designed the study as well as performed a critical revision of the article. All authors discussed the results and commented on the manuscript at all stages. All of them provided final approval of the version to be submitted.

Conflict of interest: the authors declare no potential conflict of interest.

Received for publication: 29 April 2016.

Revision received: 7 June 2016.

Accepted for publication: 7 June 2016.

(C) Copyright A. Chiereghin et al., 2016

Licensee PAGEPress, Italy

Microbiologia Medica 2016; 31:5963

doi:10.4081/mm.2016.5963

This article is distributed under the terms of the Creative Commons Attribution Noncommercial License (by-nc 4.0) which permits any noncommercial use, distribution, and reproduction in any medium, provided the original author(s) and source are credited.
MGB $^{\text {TM }}$ kit (ELITech Group). The Quality Control for Molecular Diagnostics human CMV panels was also tested.

Results: Sixteen (14\%) samples resulted negative and 59 (51.7\%) positive with a quantitative result for both assays. In the 59 samples, the coefficient of correlation was 0.856 . Bland-Altman analysis showed a mean difference of $0.11 \log _{10}$ copies/mL (standard deviation $=0.38$ $\log _{10}$ copies/mL). The assays gave CMV-DNA loads differing by $<1 \log _{10}$ DNA copies/mL in 57 samples (96.6\%) and by $<0.5 \log _{10}$ DNA copies/mL in 48 samples (81.3\%). Eleven (9.6\%) samples were positive with a quantitative result with Abbott and negative with ELITech. Sixteen (14\%) positive samples with a quantitative result for Abbott resulted positive but below the lower limit of quantification (LLQ) for ELITech. Twelve (10.5\%) samples resulted negative with ELITech and positive but below the LLQ with Abbott. No samples were positive with ELITech and negative with Abbott.

Conclusions: The assays showed a good correlation between CMVDNA levels detected and variation in CMV-DNA $<0.5 \log _{10}$ was observed in the majority of the samples. The viral load kinetic profiles of the assays were overlapping in all patients, but Abbott showed higher sensitivity in samples containing lower amount of DNA. The clinical value of this greater sensitivity requires further investigation.

\section{Introduction}

Cytomegalovirus (CMV) infection remains one of the most common complications after solid organ transplant (SOT), resulting in significant morbidity, graft loss, and occasional mortality. The adverse impact of CMV infection on graft function underscores the importance of CMV on transplant outcomes (10). Two major strategies are commonly used for prevention of CMV: universal prophylaxis and pre-emptive therapy that have resulted in significant reductions in CMV disease and CMVrelated mortality (10). Consensus guidelines for the management of CMV infection and disease in SOT recipients have been published $(10,11,16)$. These guidelines recommend the use of quantitative nucleic acid amplification (QNAT)-based assays for CMV as the main choice for diagnosis, making decisions regarding pre-emptive therapy and monitoring response to therapy $(3,4,8,9,12,17,18)$. However, currently, a universal cut-off for initiating pre-emptive therapy has not been established (10). In fact, the variability in the performance of different NAT-based assays has been documented and makes it difficult to compare viral load measurements between different laboratories and to develop uniform treatments strategies $(14,19)$. These studies 
have highlighted the need for an internationally accepted reference standard for CMV that has been recently developed $\left(1^{\text {st }}\right.$ WHO International Standard for human CMV Nucleic Acid Amplification Techniques) (6). In this study, we evaluated the performances of two commercial available RealTime polymerase chain reaction (PCR) assays - Abbott real-time CMV PCR Kit (Abbott Molecular Inc., Des Plaines, IL, USA) and CMV ELITe MGB ${ }^{\text {TM }}$ kit (ELITech Group, Milano, Italy) - for CMV DNA quantitation in whole blood (WB) samples collected by SOT recipients.

\section{Materials and Methods}

\section{Clinical samples and artificial standards}

A total of 114 serial WB specimens were included in the study. These samples were obtained from 14 selected patients with active CMV infection who had undergone liver $(n=4)$, heart $(n=5)$ and kidney $(n=5)$ transplant at our institution between July 2010 and June 2011. In particular, $35 \mathrm{WB}$ samples were collected from liver, 41 from heart and 38 from kidney transplant recipients; the median number of WB samples analysed per patient was equal to 7 (range, 4-13). For all patients, the entire episode of viral replication, preceded and/or followed by at least one WB sample resulted CMV DNA negative with PCR assay routinely used, was analysed. All patients were adults, with a male:female ratio of 9:5 and a mean age of 53 years. Paired CMV serostat uses of Donors (D) and Recipients (R) were $D+/ R+$ in 9 cases, D+/R- in 3 cases, D-/R+ and D-/R- in 1 case, respectively. For these patients, routine follow-up of CMV infection was performed using ELITech PCR assay and peripheral blood samples were collected every week during the first 3 months, then monthly until the sixth month and then every 3 months until one year post-transplant. Additional periphe ral blood samples were taken if clinically indicated. With regard to the clinical management of CMV infection, the following were used: i) prophylaxis therapy with oral valganciclovir (450 mg $\times 2 /$ day; $n=7$ ); ii) pre-emptive therapy with oral valganciclovir (900 $\mathrm{mg} \times 2 /$ day; $\mathrm{n}=8)$ or intravenous ganciclovir (10 $\mathrm{mg} / \mathrm{kg} / \mathrm{day} ; \mathrm{n}=1$ ) on the basis of 10,000 CMV DNA copies/mL WB for all the type of SOT; and iii) symptomatic therapy with oral valganciclovir $(900 \mathrm{mg} \times 2 /$ day; $\mathrm{n}=3$ ).

To compare the two real-time PCR assays, the selected samples stored at $-80^{\circ} \mathrm{C}$ were retrospectively tested using the Abbott realtime CMV PCR Kit. The 2010 quality control for molecular diagnostics (QCMD) human CMV panels (Glasgow, Scotland) were also tested by both real-time PCR assays. This panel consisted of nine samples containing various concentrations of CMV strain AD169 in either plasma or in Virus Transport Medium and one plasma sample negative for CMV.

\section{Cytomegalovirus DNA quantitation using CMV ELITe MGB ${ }^{\text {TM }}$ kit}

DNA was extracted from $200 \mu \mathrm{L}$ of ethylenediaminetetraacetic acid (EDTA)-anticoagulated WB using the QIASymphony SP instrument (Qiagen GmbH, Hilden, Germany) according to the manufacturer's instructions and eluted in $90 \mu \mathrm{L}$. An aliquot of $20 \mu \mathrm{L}$ of these extracted DNA samples was used for the CMV quantitative real-time PCR on the ABI Prism 7300 RealTime PCR system (PE Applied Biosystem, Foster City, Calif, United States). The same extraction and amplification protocol was used for QCMD panels for human CMV 2010. Primers and probes are located in the highly conserved major immediate early antigen region of the CMV genome. The lower limit of quantification (LLQ) was 225 copies/mL WB; CMV load was reported as numbers of copies $/ \mathrm{mL}$.

\section{Cytomegalovirus DNA quantitation using Abbott RealTime CMV PCR Kit}

DNA extraction was performed by Abbott mSample Preparation System DNA kit on the m2000sp instrument coupled with the Abbott CMV PCR kit. DNA was extracted from $300 \mu \mathrm{L}$ of EDTA-anticoagulated WB and eluted in $250 \mu \mathrm{L}$. Afterwards, $35 \mu \mathrm{L}$ of the eluted samples was tested using the Abbott $m 2000$ rt instrument according to the manufacturer's instructions. The same extraction and amplification protocol was used for the 2010 QCMD human CMV panels. The Abbott real-time CMV assay targets two short sequences within the UL34 and UL80.5 genes of the CMV genome. The LLQ was 40 copies/mL WB; CMV load was reported as numbers of copies/mL.

\section{Statistical techniques}

The data (in copies/mL) were $\log _{10}$ transformed prior to analysis. Quantitative correlations between the CMV DNA loads obtained using the two methods were evaluated using the Spearman correlation test. The method of Bland and Altman was used to assess the agreement between CMV DNA loads measured using the two assays.

\section{Results}

\section{Quality control for molecular diagnostics panel}

The 2010 QCMD human CMV panel was used as standard. Each sample was reconstituted by adding $1 \mathrm{~mL}$ sterile water as indicated by manufactures guidelines. CMV DNA copies obtained with ELITech and Abbott PCR assays were compared with the consensus values. The results of the evaluation are reported in Table 1. The negative QCMD control sample was negative in both assay; 1 of the two detected sample (QCMD 10-02, 230 copies/mL) was detected as negative by ELITech assay and except for this result, the values obtained with both assays were within the standard error interval of the consensus values and differed less than $0.5 \log _{10}$ copies/mL from these reference values.

\section{Clinical samples}

A total of 114 serial WB samples collected from 14 selected SOT recipients during episodes of active $\mathrm{CMV}$ infection were processed by the two assays. CMV DNA was detected by both assays in 59/114 samples (51.7\%), while results were negative in both assays for $16 / 114$ samples (14\%). Eleven out of 114 samples (9.6\%) tested positive with a quantitative result in the Abbott PCR assay however the results were negative in the ELITech PCR assay. In addition, 16 (14\%) positive samples with a quantitative result for Abbott PCR assay resulted positive but below the LLQ for ELITech PCR assay. Furthermore, 12 samples (10.5\%) were CMV DNA positive but below the LLQ with the Abbott assay and were negative with ELITech. No samples were positive in the ELITech PCR assay and negative in the Abbott PCR assay. These results are reported in Table 2 .

In the 59 samples with quantitative results by both assays, the coefficient of correlation was equal to 0.856 (Figure 1).

Bland-Altman analysis showed a mean difference (ELITech-Abbott) of $0.11 \log _{10}$ copies $/ \mathrm{mL}$ (standard deviation=0.38 $\log _{10}$ copies $/ \mathrm{mL}$ ). Moreover, ELITech and Abbott PCR assays gave CMV DNA loads differing by less than $1 \log _{10}$ DNA copies/mL in 57/59 samples (96.6\%) and by less than $0.5 \log _{10}$ DNA copies/mL in 48/59 samples (81.3\%) (Figure 2).

\section{Clinical follow-up}

Virological monitoring was performed in all 14 SOT recipients for an average of 147 days post-transplantation (range, 53-322). The performance of the two assays was evaluated in a clinical context. Patients' 
Table 1. Quality Control for Molecular Diagnostics 2010 human cytomegalovirus DNA panel evaluation.

\begin{tabular}{|c|c|c|c|c|c|c|c|c|c|}
\hline Sample & $\begin{array}{l}\text { Sample } \\
\text { content }\end{array}$ & $\begin{array}{c}\text { Sample } \\
\text { matrix } \\
\text { Mean }\end{array}$ & $\begin{array}{c}\text { Sample } \\
\text { status } \\
\text { SD }\end{array}$ & \multicolumn{2}{|c|}{$\begin{array}{l}\text { Consensus values } \\
\log _{10} \text { copies/mL }\end{array}$} & $\begin{array}{l}\text { Abbott assay } \\
\log _{10} \text { copies/mL }\end{array}$ & Delta & $\begin{array}{l}\text { ELITech assay } \\
\log _{10} \text { copies/mL }\end{array}$ & Delta \\
\hline QCMD 10-06 & CMV (AD169) & VTM & Frequently detected & 6.41 & 0.44 & 6.32 & 0.09 & 6.61 & 0.2 \\
\hline QCMD 10-03 & CMV (AD169) & VTM & Frequently detected & 5.44 & 0.45 & 5.24 & 0.20 & 5.65 & 0.21 \\
\hline QCMD 10-08 & CMV (AD169) & VTM & Frequently detected & 4.38 & 0.42 & 3.96 & 0.42 & 4.34 & 0.04 \\
\hline QCMD 10-10 & CMV (AD169) & Plasma & Frequently detected & 4.23 & 0.43 & 4.02 & 0.21 & 4.38 & 0.15 \\
\hline QCMD 10-04 & CMV (AD169) & Plasma & Frequently detected & 3.74 & 0.44 & 3.56 & 0.18 & 3.80 & 0.06 \\
\hline QCMD 10-01 & CMV (AD169) & Plasma & Frequently detected & 3.27 & 0.40 & 3.09 & 0.18 & 2.92 & 0.30 \\
\hline QCMD 10-07 & CMV (AD169) & Plasma & Frequently detected & 3.26 & 0.44 & 3.05 & 0.21 & 3.25 & 0.01 \\
\hline QCMD 10-09 & CMV (AD169) & Plasma & Detected & 2.84 & 0.46 & 2.66 & 0.18 & 2.69 & 0.15 \\
\hline QCMD 10-02 & CMV (AD169) & Plasma & Detected & 2.36 & 0.45 & 1.95 & 0.41 & neg & 2.36 \\
\hline QCMD 10-05 & CMV Negative & Plasma & Negative & neg & - & neg & neg & neg & neg \\
\hline
\end{tabular}

CMV, cytomegalovirus; VTM, Virus Transport Medium; SD, Standard Deviation; neg, negative.

Table 2. Detection of cytomegalovirus DNA in 114 whole blood samples from 14 solid organ transplant recipients using ELITech and Abbott polymerase chain reaction (PCR) assays.

\begin{tabular}{lcccc} 
Abbott PCR & \multicolumn{4}{c}{ EllTech PCR assay copies/mL } \\
assay copies/mL & Negative & $P<225$ & $P \geq 225$ & Total \\
Negative & 16 & 0 & 0 & 16 \\
$\mathrm{P}<40$ & 12 & 0 & 0 & 12 \\
\hline $\mathrm{P} \geq 40$ & 11 & 16 & 59 & 86 \\
Total & 39 & 16 & 59 & 114 \\
\hline
\end{tabular}

clinical status and therapeutic strategies were taken in consideration for the interpretation of the results. In Figure 3, we reported three representative patients of each type of SOT involved. In particular, two episodes of symptomatic CMV reactivation developed during the intermediate (2-6 months) and early ( $<1$ month) post-transplant period in one heart and kidney transplant recipient (Figure $3 \mathrm{~A}, \mathrm{C}$ ) and a primary CMV infection in a liver transplant recipient (Figure 3B) were illustrated. By analysing these patients it is possible to observe that the viral load kinetic profiles of the two assays resulted overlapping and that the Abbott assay showed higher sensitivity in samples containing lower amount of DNA (Figure 3B,C).

\section{Patient 1}

A 59-year-old male received a heart transplant for ischemic dilated cardiomyopathy in July 2010. The CMV constellation was D+/R+ and the patient received prophylactic valganciclovir $(450 \mathrm{mg} \times 2 /$ day $)$ for 1 month post-transplant. The patient developed symptomatic CMV infection (CMV syndrome) at 163 days post-transplant. CMV infection was successfully treated with oral valganciclovir $(900 \mathrm{mg} \times 2 /$ day $)$. The kinetics of CMV infection showed overlapping patterns with both assay (Figure 3A).

\section{Patient 2}

A 57-year-old female received a liver transplant for HCV-associated hepatocellular carcinoma in September 2010. CMV serostatus of donor and recipient was D+/R-. The patient received prophylactic valganciclovir (450 mg $\times 2 /$ day) for 3 months post-transplant; one month later, she seroconverted for CMV without the onset of clinical symptom due

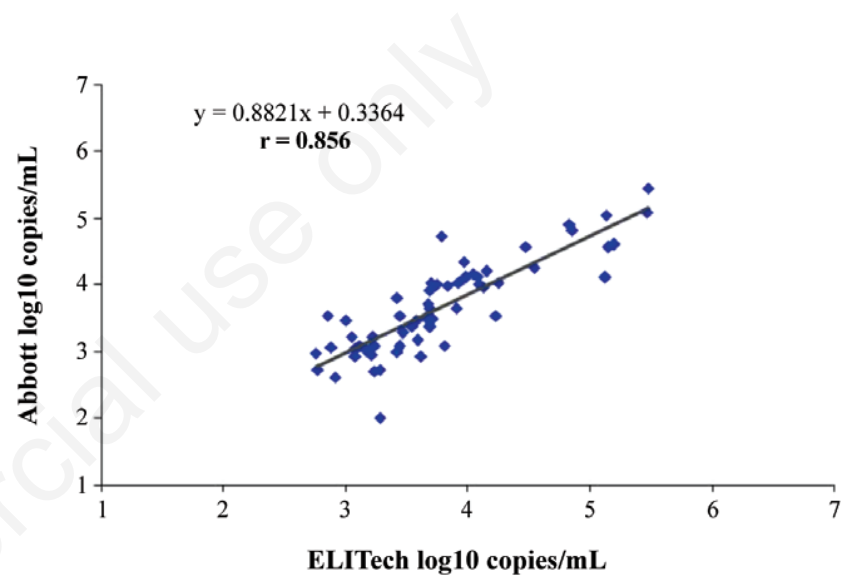

Figure 1. Correlation between whole blood samples $(n=59)$ tested positive with a quantitative result by both ELITech and Abbott polymerase chain reaction assays.

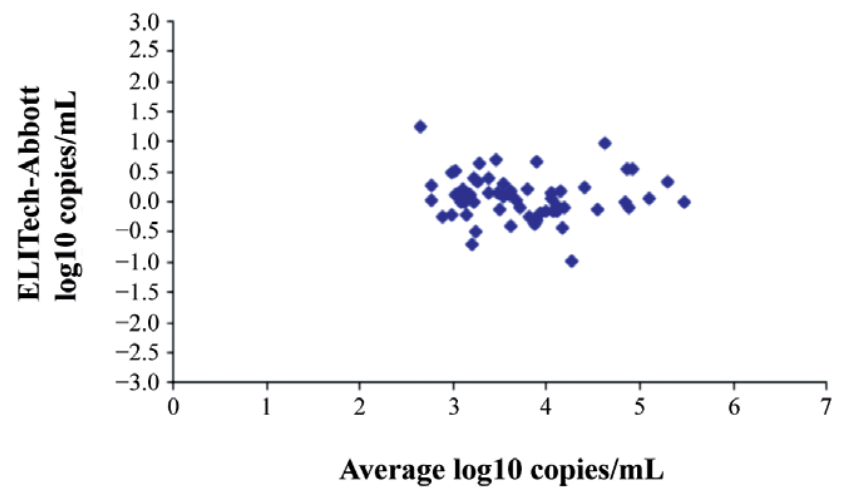

Figure 2. Bland-Altman analysis on 59 whole blood samples tested positive with a quantitative result by both ELITech and Abbott polymerase chain reaction assays. 
to CMV infection. She then received oral valganciclovir $(900 \mathrm{mg}$ $\times 2 /$ day) and pre-emptive therapy was stopped after two consecutive negative results obtained with ELITech assay. Figure 3B summarizes treatment and the kinetics of CMV infection starting 105 days after transplantation. During follow-up, the kinetics of CMV DNA were comparable for the two assays; however, the last two samples (collected at 209 and at 225 days post-transplant) were CMV DNA negative with ELITech and were positive but below the LLQ with the Abbott assay.

\section{Patient 3}

A 50-year-old male received a kidney transplant for glomerulonephritis in January 2011. The recipient and his respective donor were seropositive for CMV at the time of transplantation. As showed in Figure
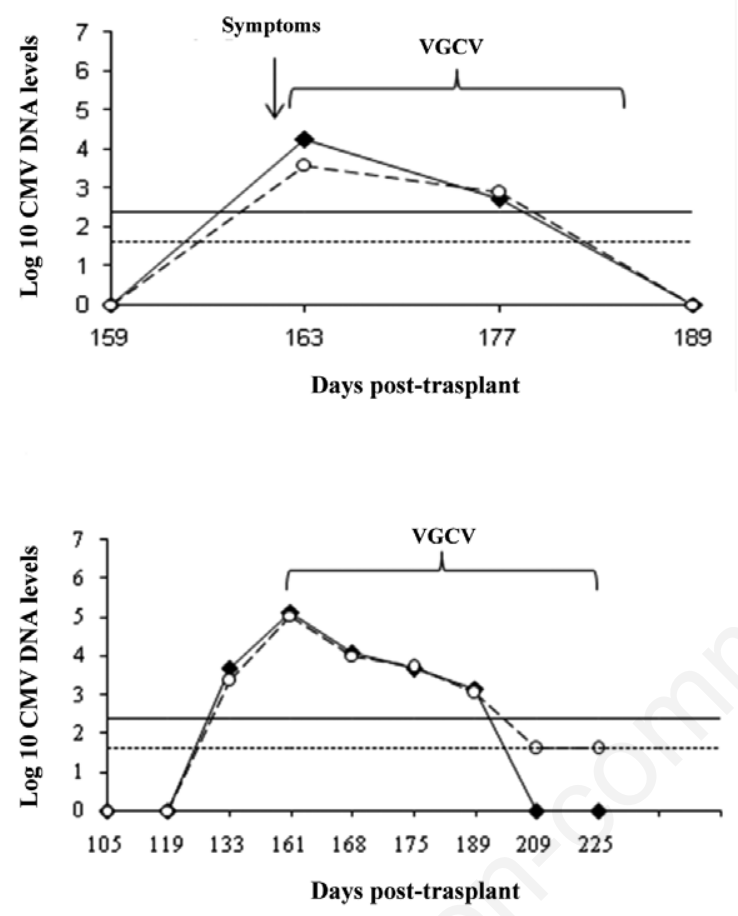

$\mathbf{B}$
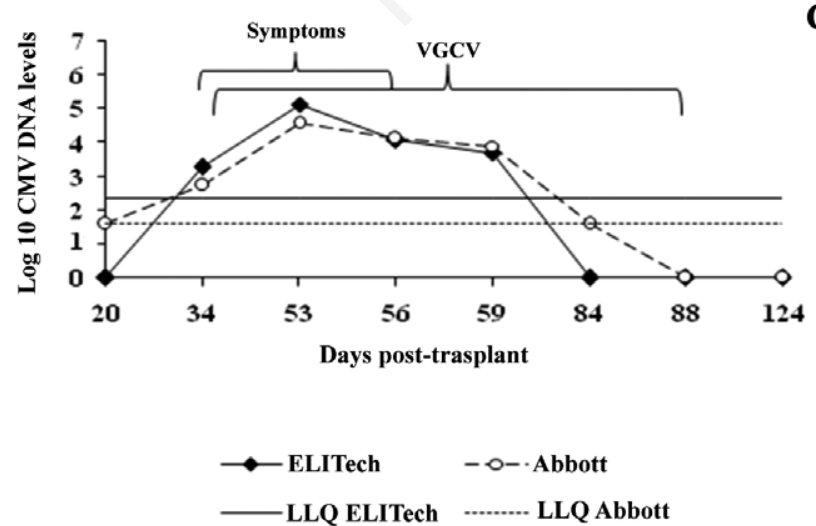

Figure 3. Kinetics of cytomegalovirus infection in 3 solid organ transplant recipients as determined by ELITech and Abbott assays (LLQ, lower limit of quantification; VGCV, valganciclovir). A) Patient 1; B) patient 2; C) patient 3.
3C, the patient developed symptomatic CMV infection (fever and neutropenia) at 34 days post-transplant; CMV infection was treated with oral valganciclovir (900 $\mathrm{mg} \times 2 /$ day) and antiviral therapy was stopped after two consecutive negative results obtained with ELITech assay.

Quantitation of CMV DNAemia by both assays starts 20 days posttransplantation. The kinetics of CMV DNA increase were comparable for the two assays, as well as the kinetics of CMV DNA decrease in response to CMV treatment. Discordant results (CMV DNA negative with ELITech and positive but below the LLQ with the Abbott assay) were restricted to the initial and final sampling times of CMV infection (day 20 and 84).

\section{Discussion}

CMV infection is an important cause of morbidity and mortality in solid organ transplant recipients, with up to $75 \%$ of patients developing or reactivating infection after transplantation (5). Significant advances have been made in the management of post-transplant CMV infection (10). The success of CMV prevention during the early post-transplant period and the improvement of CMV disease management are partly attributed to advances in diagnostic virology $(4,15,16)$. Over the years, quantification of CMV load in blood, mostly by real-time PCR, has become a mainstay of clinical management (1). Nevertheless to date, consensus CMV DNA cut-off values for clinical decision-making, particularly for pre-emptive strategies have not yet been defined, mainly due to the lack of real-time standardization (7).

In this study, the 2010 QCMD human CMV panels and a total of 114 serial WB samples collected from 14 selected SOT recipients (5 heart, 5 kidney and 4 liver) during episodes of active CMV infection were processed by two commercial available real-time PCR assays, i.e. Abbott and ELITech PCR assays.

The control panel results indicate that the specificity of Abbott and ELITech PCR assays was $100 \%$ and the sensitivity was $100 \%$ for CMV DNA levels upper to approximately 600 copies/mL (QCMD 10-09, 2.84 $\log _{10}$ copies/mL). Moreover, for the positive samples the two assays provided results less than $0.5 \log _{10}$ copies/mL difference from the consensus. Therefore, QCMD results showed robustness and accuracy of the two assays.

Regarding the comparison of ELITech and Abbott assays in clinical samples, the two assays showed a good correlation between the samples with measurable DNAemia results. Moreover, CMV DNA quantification in the majority of samples (81.3\%) showed a difference $<0.5$ $\log _{10}$. These results confirm the trend suggested by other studies $(13,14)$, according to which $0.5 \log _{10}$ DNA copies/mL represents the upper limit for divergence from the expected reference values for adequate interlaboratory comparisons of CMV DNA loads with clinical purposes. Most likely, viral load differences of $<0.5 \log _{10}$ when comparing different assays may not be considered clinically relevant and may reflect the intrinsic PCR assay variability $(2,7)$. Furthermore, the viral load kinetic profiles of the two assays overlapped in all 14 SOT recipients, but the Abbott assay showed higher sensitivity in samples containing lower amount of DNA. The clinical value of this greater sensitivity therefore requires further investigation.

\section{Conclusions}

In conclusion, the recent development of automated nucleic acid extraction devices together with the introduction of real-time technology, and the WHO International Standard for human CMV Nucleic Acid Amplification Techniques (WHO Standard) should allow researches to 
work out the equivalencies between CMV DNA loads measured by different QNAT-based assays, ultimately permitting the establishment of clinically safe CMV DNA thresholds triggering therapeutic intervention. In fact, definition of universal clinically validated thresholds for initiating pre-emptive treatment in solid organ transplant recipients is a major goal in the transplantation setting.

\section{References}

1. Atkinson C, Emery C. Cytomegalovirus quantification: where to next in optimising patient management? J Clin Virol 2011;51:223-8.

2. Caliendo AM, Schuurman R, Yen-Lieberman B, et al. Comparison of quantitative and qualitative PCR assays for cytomegalovirus DNA in plasma. J Clin Microbiol 2001;39:1334-8.

3. Caliendo AM, St George K, Allega J, et al. Distinguishing cytomegalovirus (CMV) infection and disease with CMV nucleic acid assays. J Clin Microbiol 2002;40:1581-6.

4. Emery VC, Sabin CA, Cope AV, et al. Application of viral-load kinetics to identify patients who develop cytomegalovirus disease after transplantation. Lancet 2000;355:2032-6.

5. Fishman JA, Emery V, Freeman R, et al. Cytomegalovirus in transplantation-challenging the status quo. Clin Transplant 2007;21:149-58.

6. Freyer JF, Heath AB, Anderson R, et al. Collaborative study to evaluate the proposed 1st WHO International Standard for human cytomegalovirus (HCMV) for nucleic acid amplification (NAT)based assays. Geneva: WHO/BS/10.2138; 2010. pp 1-40.

7. Furione M, Rognoni V, Ermanna C, Baldanti F. Kinetics of human cytomegalovirus (HCMV) DNAemia in transplanted patients expressed in international units as determined with the Abbott Real Time CMV assay and an in-house assay. J Clin Virol 2012; 55:317-22.

8. Humar A, Gregson D, Caliendo AM, et al. Clinical utility of quantitative cytomegalovirus viral load determination for predicting cytomegalovirus disease in liver transplant recipients. Transplantation 1999;68:1305-11.

9. Humar A, Kumar D, Boivin G, et al. Cytomegalovirus (CMV) virus load kinetics to predict recurrent disease in solid organ transplant patients with CMV disease. J Infect Dis 2002;186:829-33.

10. Kotton CN, Kumar D, Caliendo AM, et al. International consensus guidelines on the management of cytomegalovirus in solid organ transplantation. Transplantation 2010;89:779-95.

11. Kotton CN, Kumar D, Caliendo AM, et al. Transplantation Society International CMV Consensus Group. Updated international consensus guidelines on the management of cytomegalovirus in solidorgan transplantation. Transplantation 2013;96:333-60.

12. Lao WC, Lee D, Burroughs A, et al. Use of polymerase chain reaction to provide prognostic information of human cytomegalovirus disease after liver transplantation. J Med Virol 1997;51:152-8.

13. Pang XL, Chui L, Fenton J, et al. Comparison of LightCycler-based PCR, COBAS Amplicor CMV monitor, and pp65 antigenemia assays for quantitative measurement of cytomegalovirus viral load in peripheral blood specimens from patients after solid organ transplantation. J Clin Microbiol 2003;4:3167-74.

14. Pang XL, Fox JD, Fenton JM, et al. Interlaboratory comparison of cytomegalovirus viral load assays. Am J Transplant 2009; 9:258-68.

15. Piiparinen H, Lautenschlager I. Quantitative TaqMan assay for the detection and monitoring of cytomegalovirus infection in organ transplant patients. Methods Mol Biol. 2006;335:147-56.

16. Razonable RR, Emery VC; 11th Annual Meeting of the IHMF (International Herpes Management Forum). Management of CMV infection and disease in transplant patients. 27-29 February 2004. Herpes 2004;11:77-86.

17. Razonable RR, van Cruijsen H, Brown RA, et al. Dynamics of cytomegalovirus replication during preemptive therapy with oral ganciclovir. J Infect Dis 2003;187:1801-8.

18. Rollag H, Sagedal S, Kristiansen KI, et al. Cytomegalovirus DNA concentration in plasma predicts development of cytomegalovirus disease in kidney transplant recipients. Clin Microbiol Infect 2002;8:431-4.

19. Wolff DJ, Heaney DL, Neuwald PD, et al. Multi-Site PCR-based CMV viral load assessment-assays demonstrate linearity and precision, but lack numeric standardization: a report of the association for molecular pathology. J Mol Diagn 2009;11:87-92. 